\title{
Reality check for organoids in neuroscience
}

\author{
To better study human neurodevelopment, researchers advance organoids as brain models.
}

\author{
Vivien Marx
}

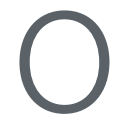

rganoids, which are stem-cellderived three-dimensional experimental models, are making inroads into many fields, including neuroscience, where there's a dire need for models of complex processes such as in utero neurodevelopment and neuropsychiatric disorders ${ }^{1-4}$. But brain organoids aren't exactly getting a smooth ride into neuroscience. "From my vantage point, brain organoids are way cool models of themselves," says Stanford University neuroscientist Carla Shatz. As with all in vitro studies, she says, "even under the more realistic conditions of three dimensions rather than in flatland, whatever you learn tells you about what can happen, not what does happen in vivo." These models can teach a lot about the cell biology of human neurons, "which is wonderful," she says. "Then the question is, how to reality-test findings using human brain samples?"

Undoubtedly, say brain organoid methods developers, these are not 'brains in a dish' nor windows into all facets of in utero neurodevelopment, nor do they reveal every detail of how a newborn's brain becomes the brain of a little person with a case of the 'terrible twos' and a resolute command of the word "no." As Madeline Lancaster from the MRC Laboratory of Molecular Biology says, "all the brain organoids in vitro so far lack many of the important features of the brain in vivo," such as a functional vascular system and an immune system. Organoids lack the anatomy of the real human brain, says Giorgia Quadrato from University of Southern California's Keck School of Medicine. But, she says, they are models that let us characterize human brain development and disease in ways that have eluded the neuroscience community for decades. To grow their research community and address the concerns of those in neuroscience who might view organoids and conclusions drawn from them with furrowed brow, Lancaster, Quadrato and others are doubling down on advancing organoid methods.

\section{Second-gen organoids}

Brain organoids are becoming more complex and dynamic. The Lancaster lab

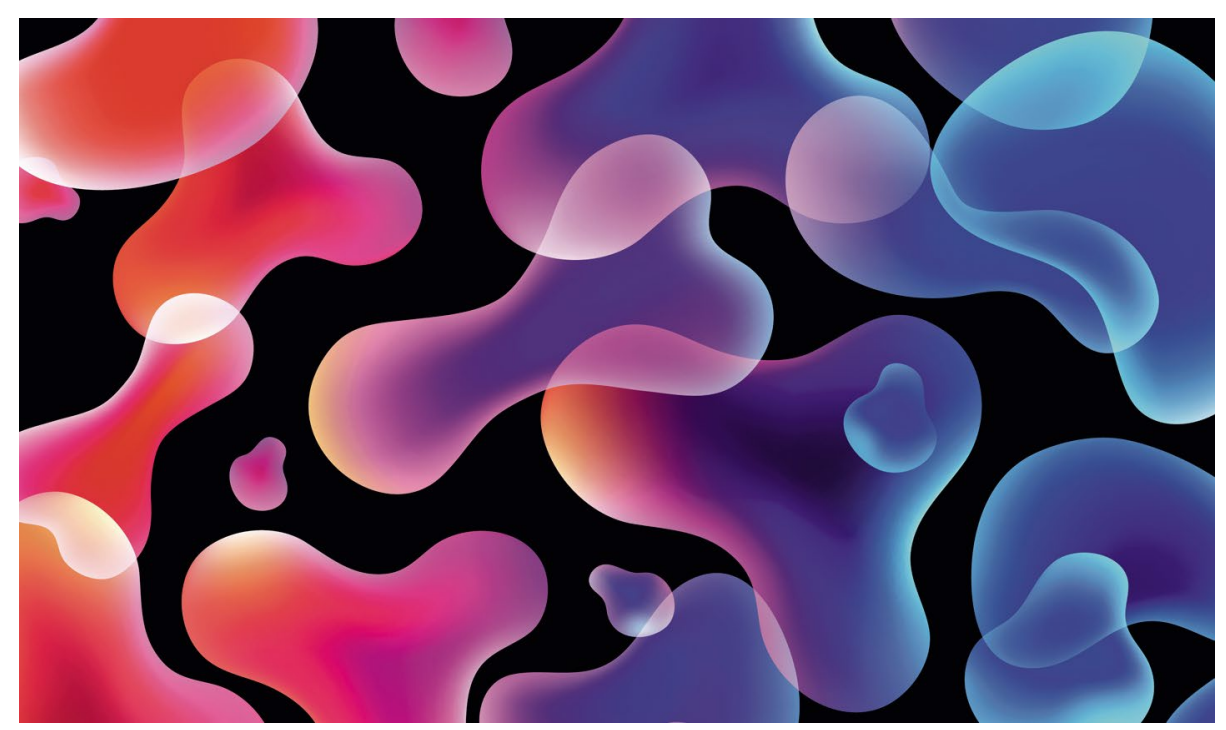

Brain organoids grow as floaty blobs (here an artist's impression). They serve to model aspects of human brain development intractable by other means. Credit: a-r-t-i-s-t / DigitalVision Vectors / Getty

has generated organoids from induced pluripotent stem cells (iPSCs) that can secrete a fluid akin to cerebrospinal fluid $(\mathrm{CSF})^{5}$. It's a model of the brain's choroid plexus (ChP), which is found in each brain ventricle and secretes CSF. Choroidal epithelial cells regulate what passes from blood to CSF. As Violeta Silva-Vargas and Fiona Doetsch of the University of Basel note $^{6}$, "a lack of tools has limited exploration of the ChP, especially in humans." From a proteomics perspective, says Lancaster, it's hard to tell the difference between the fluid these organoids make and the brain's actual CSF. "But we cannot really call it actual CSF because it is made in vitro and there are things coming from the media, such as mouse- or cow-derived proteins, that of course would not be present in vivo in human CSF," she says. Cow albumin, and not human albumin, is in this system because bovine serum albumin is present as a cell medium additive. Since publication, she has heard from labs who plan to use the system for neurotoxicology testing to check for unwanted crossing into the brain. Others teams plan on exploring better ways to get drugs into the brain or how CSF might change in disease conditions.
The Lancaster lab is using the organoids to study development and evolution of the choroid plexus, also in relation to the rest of the brain. "We are also finding that these organoids provide an interesting window into the biology of this understudied brain region, and that it can also be used in conjunction with other brain organoids to understand disease biology, including the effects of SARS-CoV-2," she says.

As a medical student in Rumania, Stanford University researcher Sergiu Paşca wanted improvement for his patients suffering from neuropsychiatric disorders - certainly not by using trepanning, in which a hole is bored into a person's skull, to remove a 'madness stone', as was performed in the Middle Ages and depicted by Hieronymus Bosch, one of Pașca’s favorite painters. In the summers, Pașca worked in the labs of the Max Planck Institute for Brain Research in Frankfurt and recorded data from the cat visual cortex. "I was astounded what it actually means to have access to a neuron," he says. He dreamt of directly studying human neurons. When ways emerged to generate iPSCs, he jumped into a research career as a postdoctoral fellow in Ricardo Dolmetsch's lab at 


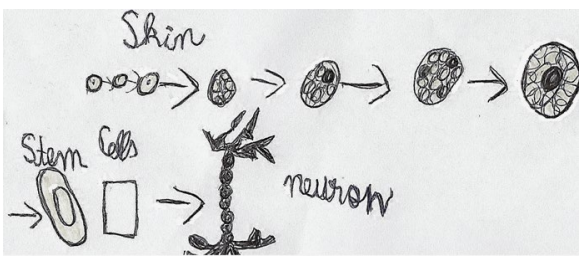

To model aspects of autism, skin cells are induced into stem cells, neurons and 3D organoids. Sketch by Sergiu Pașca's patient E.D., who has autism spectrum disorder. Credit: E.D.

Stanford. He modeled a mutation linked to autism that affects calcium channels in electrically active neurons derived from iPSCs. Plating neurons in monolayers and keeping them alive to observe long enough to model cortical development was "a nightmare," he says. Frustration led him to try a low-attachment plate where the cells grew into floating, spherical structures. Now in his own Stanford lab, he leads a team that has advanced these techniques of guiding organoids that live for up to 800 days. Organoids are a way to scale up experiments. They can be assessed at high resolution with single-cell analysis. Applying molecular biology techniques to organoids is a way to get at the human-specific nature of neuropsychiatric disorders and brain development. The Pașca lab and researchers elsewhere have linked organoids into assembloids. One such assembloid involves an organoid enriched for excitatory and another enriched for inhibitory neurons ${ }^{7}$. The model captures aspects of axonal pathfinding and also migration, such as how some populations of neurons migrate from ventral to dorsal forebrain. This migration occurs in the brain of a fetus and continues postnatally, says Pașca and it appears to be in disarray in a number of neurodevelopmental conditions, including some types of epilepsy and autism. The lab is developing a three-way cortico-motor assembloid. It's a cortical organoid, a spinal cord organoid and a muscle grown in vitro into a three-dimensional structure. In the body, cortical neurons project to the spinal cord and connect to spinal motor neurons, which project to muscle and form neuromuscular junctions that can trigger muscle activity. In this circuit model, optogenetic stimulation of the cortical organoid makes the muscle organoid twitch.

\section{A question of identity}

When making brain organoids, researchers choose from many protocols and tweak them. For her latest project, Lancaster modified the lab's existing protocol for making cerebral organoids. "In fact, we found that various approaches for generating a forebrain identity work fine as a starting point," she says. That might involve a cerebral organoid kit such as the commercial one from Stemcell Technologies, she says, or homemade media and a scaffold of fibers made of polylactide-co-glycolide as her lab has done previously ${ }^{8}$. To promote a choroid plexus subregional identity, says Lancaster, it was key to use Wnt and Bmp pathway activators to help direct the tissue to a more dorsal identity, reflecting the area where the choroid plexus develops. Although a simple tweak, the "difficult part was figuring out the correct concentration and timing, but once we figured that out, we found this little nudge is highly efficient."

As USC's Quadrato says, vexing conditions such as autism spectrum disorder, bipolar disorder or schizophrenia have an array of symptoms that can vary considerably between individuals. The conditions are mainly polygenic, with heterogeneous combinations of many alleles acting together, and various anatomic and circuit changes may also play a role. Every patient might have a different genetic background. To model mechanisms underlying these disorders, one can start with a patient's cells, induce them to become pluripotent stem cells, generate organoids from them and perform comprehensive characterization, including single-cell RNA sequencing. Cortical organoids have become more robust and reproducible, says Quadrato, thanks to work by a number of teams, including Paola Arlotta's lab at Harvard University ${ }^{9,10}$. (Quadrato completed her postdoctoral fellowship in the Arlotta lab but did not lead the work in question.) The Arlotta team and colleagues at other institutions applied single-cell RNA sequencing to characterize cells from 21 organoids collected at three and six months. Cortical organoids from different human stem cell lines, both male and female, delivered almost identical compendia of cortical cell types. "You always get the same ratio of different cortical cell types in each individual organoid," says Quadrato. Identity matters right at the start of brain organoid experiments. Stem cells grow into balls of cells called embryoid bodies, which can potentially differentiate into the three germ layers - ectoderm, mesoderm and endoderm - that give rise to all the human body's tissues. Brain organoid researchers nudge this development. They can use signaling molecules such as morphogens to generate ectoderm, the layer from which the brain develops. "The default choice of the organoid is to become cortex," says Quadrato. Lineage trajectories between human cortex and cortical organoids are similar. Some cellular subtypes can be missing in an organoid, which will not have all brain regions or sensory input from a 'body'. Although cortical organoids have become quite reproducible, "if you want to make other brain regions, then it becomes more difficult," says Quadrato. The cerebellum holds her fascination as the brain structure with the most neurons in the brain. It has expanded the most over the course of evolution and appears to have played a big role in the acquisition of human-specific cognitive traits. Evidence is growing about its role in conditions such as autism. One of her lab's projects is assuring that cerebellar organoids can be made reproducibly. A brain organoid will always have a tendency to make some forebrain, she says. That means organoids of other brain regions can vary from one batch to another and across different cell lines. This heterogeneity and variability can make it difficult for scientists to draw conclusions from their

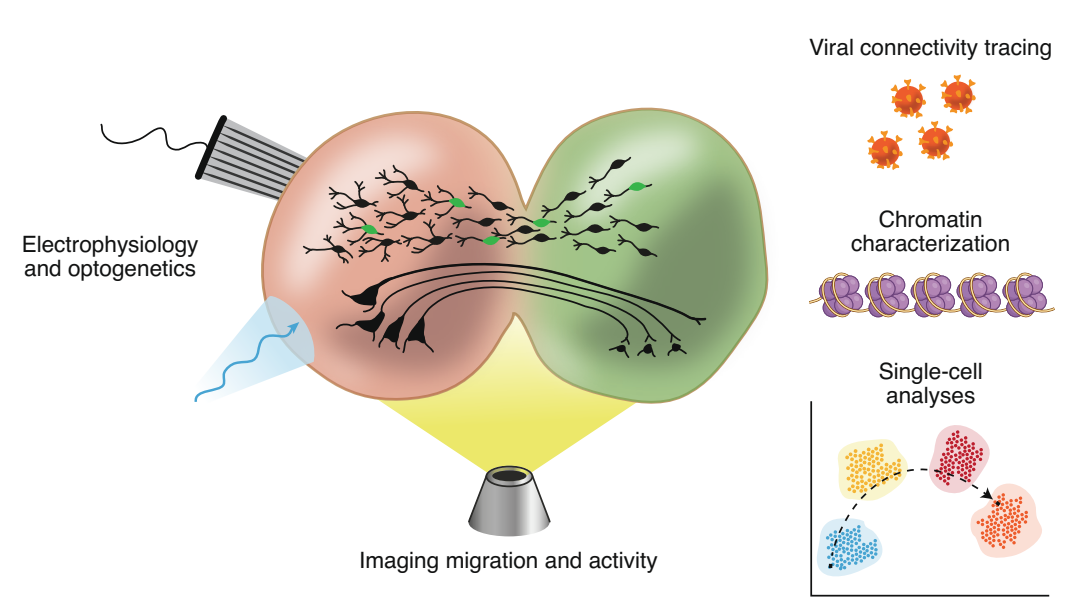

The Pașca lab has developed assembloids, which combine different organoid types. Organoids can be characterized with many techniques. Credit: Adapted with permission from ref. ${ }^{3}$, Springer Nature 
organoids about the mechanisms underlying disorders. High-throughput, high-resolution techniques are needed to reveal the identity of cells in a given organoid; "otherwise, it's very difficult to understand what's going on and to characterize them," she says. She is concerned that some labs start working with organoids but underestimate how much time, effort and, ultimately, money, it takes to thoroughly characterize organoids. Costs will drop as technologies mature, but, using the wrong techniques or applying them to an in vitro model in the wrong way, "you can draw conclusions that are completely wrong," she says. Among the techniques she is establishing in her lab is Patch-seq, which involves recording electrical activity from a neuron or groups of neurons through patch-clamping followed by RNA sequencing. When working with mice, location hints at the cell types one is recording from. An organoid lacks such anatomical reference points, and 'blind' recording from just anywhere in an organoid is a bad idea, she says. With Patch-seq, one can aspirate the RNA from the just patch-clamped cells and "then you look at that profile; it's very helpful."

\section{Growing the family}

"You can really resolve at the cell type level what's going on," says Michael Fernando about Patch-seq. He is a PhD student at Icahn School of Medicine at Mt. Sinai in the lab of Kristen Brennand, who models neuropsychiatric disease by merging stem cell biology and neuroscience and who has begun to use organoids. He is co-advised by Mt. Sinai neuroscientist and electrophysiologist Paul Slesinger. Fernando wants to learn and apply Patch-seq with organoids to assess how perturbation or gene-editing experiments might affect gene expression or electrophysiology readouts. Fernando took part in a hands-on course about protocols and experimental design run by the Pașca lab at Stanford to reach out to the "next generation" of brain organoid researchers. "It was a really cool experience," says Fernando. "I was e-mailing my PIs throughout that week with new ideas." After lectures, the participants settled in at workstations. It takes weeks to differentiate stem cells into neurons and grow them into organoids, so the Pașca lab had prepared organoids for them. Beyond stem-cell culture techniques, says Fernando, they learned how to dislodge stem cells from wells to become "beautiful, spherical" balls. "They should be happy," he says. Protocols differ, such as in the use of types of growth media, and each component makes a difference. After the course, when Fernando and fellow student Aleta Murphy generated

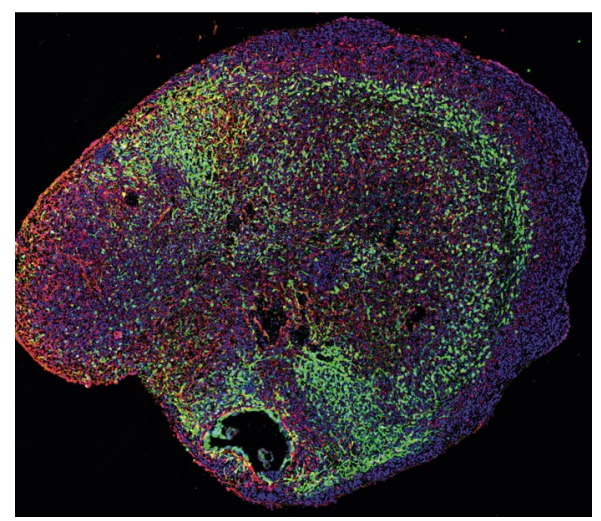

There are different types of brain organoids. Here, a cerebellar organoid from the Quadrato lab shows the dendritic marker MAP2 (red), Purkinje cell marker CALB1 (green) and cell nucleus marker DAPI (blue). Credit: A. Atamian, Quadrato Lab, USC

organoids some began to die. He asked the Pașca lab for advice through the course's Slack channel, which has now been opened to the entire Brennand lab, and "they're great at responding," he says. "When they do this again, our family will grow," he says. "We'll get to keep interacting with each other over our careers."

Fernando's $\mathrm{PhD}$ project is about using stem cell lines, genomics and electrophysiology to study the function of the neurexin-1 gene's many splice variants. Deletions in this gene have been detected in neurons differentiated from cells donated by people with a number of neuropsychiatric conditions, such as schizophrenia, autism and intellectual disability. In 2011, Brennand and others compared neurons derived from schizophrenia patients with cells from people without this condition and found diminished neuronal connectivity ${ }^{11}$. Some variants are rare, others common; they likely combine to confer disease risk. Building on such work and using organoids, Fernando wants to explore cell-type-specific effects of neurexin splicing isoforms and broader effects, since neurexins are essential to neurotransmission and neurocircuitry. Some labs have assessed mouse neurons with neurexin-1 deletions and found the neurons look unchanged, he says, yet the same deletions in human neurons generate distinct phenotypes. With human brain organoids, "we're getting closer to a better model system," he says. As a young neuroscientist he has already learned how important it is to heed species differences. With organoids "we can use a human-specific platform to evaluate these questions."
The mouse brain resembles the human brain in many ways, and mice are used to study neurodevelopment or psychiatric disease, says Quadrato, who works in mice and organoids. But the mouse and human brains are also anatomically quite distinct. For example, the mouse brain is lissencephalic, lacking the human cortex's deeply folded structure. Psychiatric disorders affect higher order brain function so it's difficult to model them in mice, she says. Studying human brain development in utero is not possible. When labs study human fetal tissue they have just a 'snapshot' at a specific time point. As a brain model, says Quadrato, organoids can capture processes of brain development and disease in new ways. To share technology with labs that have less stem cell experience, kits "will make a big difference" in standardizing and broadening efforts, says Pașca. This fall, Stemcell Technologies is launching STEMdiff Dorsal and Ventral Forebrain Organoid media systems, licensed from Stanford and developed in the Pașca lab, says Jason Hamlin, who focuses on products for neuroscience labs at the company.

\section{Of two minds}

Some experimentalists generally distrust theory, says Brandeis University neuroscientist Eve Marder. To them "theory, by definition, is not real." Theory suggests new ways of thinking rather than replicating what is known. In her view, the reactions to organoids by some in the neuroscience community share some features of this distrust of theory. It's the difference between those "who study what actually is" and people trying ways "to find out how things might be." It's "fabulous" to work in organoids in the way "really good theory is incredibly instructive and revealing in neuroscience," says Marder. Experiments are possible with organoids that are not feasible in a normally developing brain. But using organoids is "constructing something, which is not the same as the real brain." When such work is done well and the right questions are asked, insights become possible that lead to better questions to ask when working with the "real brain." Marder studies neural circuits in lobsters and crabs. One side of her "finds organoids extremely intriguing," she says. "And then the conservative, neuroscience reductionist in me still believes that they're made up." Some observations in organoids might not be terribly useful for understanding how things work in the real brain, but, she says, organoids will reveal aspects that "could open up whole new lines of investigation because you see something you never would have otherwise imagined." She is comfortable holding seemingly 
mutually inconsistent positions. Organoids reveal much about the potential of biological material and cells. "But they are not going to tell you how the actual brain did it." These models can yield insight into fundamental mechanisms involved in brain development, but the way these mechanisms are called into play during actual brain development "might be different in important, and possibly unpredictably important, ways," she says. Among the puzzles neuroscience labs face, says Marder, is discerning whether a finding is a fundamental, general principle or mainly the idiosyncrasies of a particular species, a particular brain region or group of neurons. Some labs navigate these puzzles well while others get trapped by the idiosyncrasies of their preparations. Pharmacology-oriented labs need to be especially careful. Compounds act differently in different species, in different cell types, and at different temperatures and $\mathrm{pH}$ levels. Organoids have the advantage that they can be made with human cells. "They're not a human brain, but they're human," says Marder. Although circuits can be modeled with organoids, to deeply understand a circuit one needs to work in an animal, she says. Organoids let researchers study neurodevelopment in ways that cannot be studied in people. "Is the way the organoid does it the way it happens in utero? Maybe, maybe not," she says. A lucky lab will learn "something completely new," and then they need ways to assess the finding in an actual developing brain. "The organoid is not a brain; it's not a developing baby," she says. "The real key is to force people to be scrupulously honest about what they think the new principles are that they

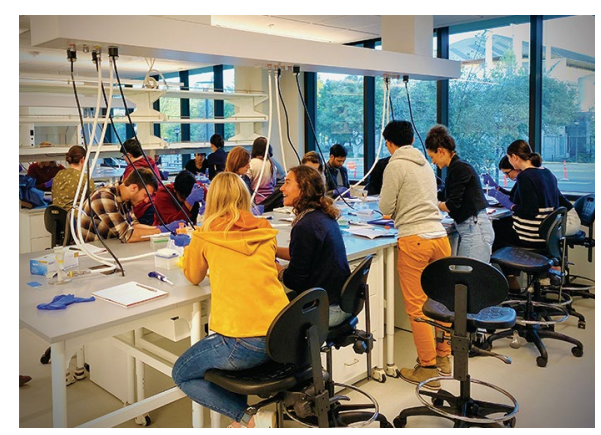

A hands-on course devoted to next-generation brain organoid researchers takes place at Pașca's lab at Stanford with participants from around the world. Credit: S. Pașca have learned," she says. Good labs studying organoids are "going to discover things that we didn't know," she says.

Organoids are still such a new area of research, says Lancaster, and "we are admittedly still just learning what these tissues can do." Her background is in vivo research and early brain development. "I am always struck by the similarities of organoids and the developing brain," she says. Researchers who are not developmental biologists might look at a brain organoid and think "'well, that doesn't really look like a brain," she says. When assessing the cells and the tissue architecture, "you realize, actually, it's truly remarkable how similar it is," she says. "Also, I think it's important to remember that even sometimes what some may call 'in vitro artifacts' can be highly informative, as they tell us what cells are capable of and reveal certain cellular behaviors that you didn't even realize were also happening in vivo."

\section{Blending, melding}

One issue Pașca sees is that the neuroscience and stem cell community often lead rather separate lives. Many common neuroscience tools such as patch-clamping and optogenetics are only slowly being integrated into work with organoids, which are a "new preparation," he says. More cross-field communication is needed to use these models to learn about the "inaccessible phases of human brain development," such as what happens in late stages of pregnancy and postnatal development.

As a developmental neurobiologist who keeps an eye on translational aspects, Quadrato sees great promise in organoids for modeling the human brain. Researchers need to check, for example, whether a particular mouse brain phenotype is also a human phenotype and understand whether changing the genetic background changes the phenotype or not. "I think, for example, combining different models of the brain is what should be done," she says. She collaborates with colleagues in tissue engineering and synthetic biology with the goal of building assembloids and controlling how axonal connections form between the organoids. Lancaster finds reproducibility with brain organoids these days "indeed much improved." Her sense is that "a melding of developmental biology and bioengineering will be an important

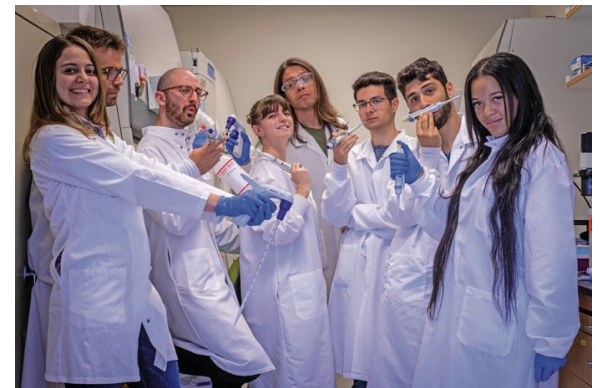

Modeling the human brain with organoids is collaborative, says USC researcher Giorgia Quadrato (left), shown here with her lab members. Credit: S. Bianco

future direction that can drive further evolution of these techniques." For example, incorporating mechanical signals as well as signaling factors "will be key in my view."

Work with brain organoids can seem daunting to newcomers, says Quadrato, but there's much room for the brain organoid research community to grow its ranks. This work will combine many techniques, including single-cell techniques, several disciplines and different ways of modeling the human brain. She sees no reason to, say, use only organoids and forget about mice and other models. Pașca agrees. Brain organoids are another tool, none of which is perfect, he says. A brain organoid is not a real human brain, says Quadrato, it's a "reductionist model." One needs, she says, to tease out the limitations of the organoid system as well as its strengths - "what we can model with high fidelity and what we can't."

Vivien Marx ${ }^{\bowtie}$
Nature Methods.
$\aleph_{\text {e-mail:v.marx@us.nature.com }}$

Published online: 9 September 2020 https://doi.org/10.1038/s41592-020-0964-z

References

1. Velasco, S., Paulsen, B. \& Arlotta, P. Annu. Rev. Neurosci. 43, 375-389 (2020).

2. Amin, N. D. \& Paşca, S. P. Neuron 100, 389-405 (2018).

3. Pașca, S. P. Nature 553, 437-445 (2018).

4. Quadrato, G., Brown, J. \& Arlotta, P. Nat. Med. 22, 1220-1228 (2016).

5. Pellegrini, L. et al. Science 369, eaaz5626 (2020).

6. Silva-Vargas, V. \& Doetsch, F. Science 369, 143-144 (2020).

7. Birey, F. et al. Nature 545, 54-59 (2017).

8. Lancaster, M. A. et al. Nat. Biotechnol. 35, 659-666 (2017).

9. Yoon, S. J. et al. Nat. Methods 16, 75-78 (2019).

10. Velasco, S. et al. Nature 570, 523-527 (2019).

11. Brennand, K. J. et al. Nature 473, 221-225 (2011). 